Rev. Psicol. (Arequipa. Univ. Catól. San Pablo) / Año 2021 / Vol 11 / Nº 2 / pp. 11-20 ISSN 2306-0565 versión impresa / ISSN 2311-7397 versión on line

\title{
Resiliencia y autoconcepto en adolescentes peruanas en situación de abandono
}

\author{
Resilience and Self-Concept in Fostered Peruvian Adolescents
}

Valeria de La Fuente-Figuerola

Forum Centro de Estudios Familiares, Cajamarca, Perú

(iD https://orcid.org/oooo-0oo2-2189-1100

Correspondencia: valeg6.dlf@gmail.com

Andy Rick Sánchez-Villena

Universidad Privada del Norte, Cajamarca, Perú

(iD) https://orcid.org/oooo-ooo1-7828-5884

Correo electrónico: andysavig2@gmail.com

Juan Pablo Sarmiento-Longo

Universidad Privada del Norte, Cajamarca, Perú

(iD) https://orcid.org/oooo-0oo2-9742-2992

Correo electrónico: sarlongo_cr@hotmail.com

\section{Resumen}

Una situación de abandono podría incrementar la probabilidad de que las adolescentes tengan una concepción negativa de ellas mismas y dificultar su manera de afrontar situaciones adversas. Por ello, el objetivo principal de este artículo es determinar la relación entre la resiliencia y el autoconcepto en adolescentes en situación de abandono. Participaron 32 adolescentes de un albergue, con edad promedio de 14 años. El autoconcepto se evaluó con la Escala de Autoconcepto Forma 5 (AF-5) y para medir resiliencia se utilizó la Escala de Resiliencia para Adolescentes (ERA). Los resultados muestran que hubo una correlación positiva y alta $(r=.789)$ entre ambas variables. Se concluye que, a mejor autoconcepto, habrá mayor nivel de resiliencia.

Palabras clave: Resiliencia, autoconcepto, adolescentes, abandono.

\section{Abstract}

Abandonment may increase the probability that adolescents have a negative self-concept and hinder their coping within adverse situations. Therefore, 
the aim of this article is to determine the relationship between resilience and self-concept in abandoned adolescents. 32 adolescents in foster care participated, with an average age of 14 years old. Self-concept was measured with the Form 5 Self-Concept Scale $(A F-5)$ and resilience was measured with the Resilience Scale for Adolescents (ERA). The results showed that resilience and self-concept had a positive and high correlation $(r=.789)$. It is concluded that a stronger self-concept, was associated with a higher level of resilience.

Keywords: Resilience, self-concept, adolescents, abandonment.

\section{Introducción}

La adolescencia es una etapa de crisis vital en el ser humano que ocurre entre los 11 y 20 años de edad, en la cual se dan cambios biológicos, psicológicos, emocionales y sociales (Papalia et al., 2009). En ella, las personas adquieren habilidades de regulación emocional (Morris et al., 2017), aprenden estrategias de afrontamiento (Chua et al., 2015) y desarrollan aspectos relevantes de la salud mental como el autoconcepto y la resiliencia (Moneta et al., 2001), debido a que enfrentan situaciones nuevas que les permite consolidar su propia identidad (Crocetti, 2017).

El autoconcepto hace referencia a percepciones, atribuciones, creencias o etiquetas que tiene una persona respecto de sí misma, las cuales pueden ser construidas a partir de la interacción con personas importantes (Crocetti et al., 2016; Shavelson et al., 1976) o del propio desempeño en el ámbito académico, social, emocional, físico (Shavelson \& Bolus, 1982), laboral y familiar (Musitu \& García, 2004).

El autoconcepto en adolescentes abandonados se relaciona con factores como la cohesión, la comunicación y la ruptura familiar (Prabandani \& Santoso, 2017); el consumo de sustancias (Oshri et al., 2017); el hurto, el vandalismo, la violencia entre pares (Cox \& Shore, 2017), la depresión (Woods et al., 2013), los pensamientos suicidas (Denton et al., 2017) y la resiliencia (Dey \& Beena Daliya, 2019; Kurniawan et al., 2018; Mishra \& Sondhi, 2019; Santoso \& Zamroni, 2017). Sin embargo, la situación de abandono no necesariamente conlleva a un pronóstico negativo, pues los adolescentes tienen la capacidad de adaptarse a las situaciones adversas (Drapeau et al., 2007; Humphreys et al., 2018; McGloin \& Widom, 2001), ello explicaría por qué algunos adolescentes institucionalizados, víctimas de situaciones traumáticas, superan estas dificultades (Edmond et al., 2006; Samuels \& Pryce, 2008; Smith et al., 2013).

La resiliencia hace referencia a «la capacidad de un sistema dinámico para resistir o recuperarse de desafíos significativos que amenazan su estabilidad, viabilidad o desarrollo» (Masten, 2011, p. 494), lo cual incluye un abanico de características protectoras como la autoestima, el autorrespeto y la autoeficacia (Rutter, 1999). La resiliencia en adolescentes abandonados se ha visto relacionada con la separación de los padres (Rowe et al., 2016), el 
maltrato familiar (Jaffee et al., 2007), el abandono familiar (Sherr et al., 2017), la soledad, la desesperanza (Rew et al., 2001), el optimismo, el afrontamiento activo (Domhardt et al., 2015) y el autoconcepto (Zolkoski \& Bullock, 2012).

Entonces, la separación del núcleo familiar y los acontecimientos de vida estresantes, pueden afectar directamente la resiliencia y el autoconcepto de los adolescentes en abandono, sin embargo, la retroalimentación positiva de los cuidadores, profesores y compañeros, junto al logro de objetivos, contribuyen a reforzar la capacidad adaptativa, la construcción de un plan de vida y la percepción de ser capaz de lidiar con las dificultades (Höjer \& Johansson, 2013; Honey et al., 2011).

En el contexto nacional, hasta el año 2011, existían alrededor de 17 ooo NNA albergados en diferentes Centros de Atención Residencial (CAR) (Defensoría del Pueblo, 2011). Datos más recientes del Ministerio de la Mujer y Poblaciones Vulnerables (Ministerio de la Mujer y Poblaciones Vulnerables [MIMP], 2014), refieren que hasta el año 2013, hubo un total de 4961 NNA en presunto estado de abandono familiar, de los cuales el 59\% fueron puestos a disposición de instituciones privadas y el $17 \%$ a instituciones públicas. Otro informe del Programa Integral Nacional para el Bienestar Familiar (Programa Integral Nacional para el Bienestar Familiar [INABIF], 2019), refiere que solo durante el mes de octubre del 2019, se atendieron 1744 NNA en los diferentes CAR del país por desprotección familiar, de los cuales $64.9 \%$ eran adolescentes y 1055 eran mujeres. A nivel regional, hasta el 2015, se reportaron 37 casos de NNA en situación de abandono o bajo circunstancias difíciles, tales como consumo de sustancias, pandillaje, fugas del hogar, entre otros (Gobierno Regional de Cajamarca [GRC], 2017).

Ante ello, la presente investigación tiene como objetivo principal analizar la relación entre la resiliencia y el autoconcepto en adolescentes en situación de abandono de un albergue peruano.

\section{Método}

\section{Participantes}

La muestra estuvo conformada por 57 adolescentes de un albergue estatal de la ciudad de Cajamarca, Perú. De las cuales, solo 32 fueron seleccionadas para este estudio, por cumplir el criterio de encontrarse en situación de abandono moral y/o material. Las participantes tenían entre 12 y 17 años de edad $(M=14.63 ; \mathrm{DE}=1.72)$. El tiempo mínimo de institucionalización fue de un mes y el máximo de 14 años. Cabe señalar que la muestra fue seleccionada de manera no probabilística, pues se tomó en cuenta el criterio mencionado anteriormente.

\section{Instrumentos}

Escala de Resiliencia para Adolescentes (ERA) (Prado \& Del Águila, 200o): Es un instrumento de autorreporte que evalúa la conducta resiliente en siete dimensiones (Insight, Independencia, Interacción, Moralidad, Humor, Iniciativa 
y Creatividad) y consta de 34 ítems, estos se miden en una escala Likert de cuatro puntos, que van desde $1=$ "Rara vez" hasta 4= "Siempre". La validez fue hallada a través de análisis factorial y la confiabilidad fue de $\alpha=.862$.

Escala de Autoconcepto Forma 5 (AF-5) (García \& Musitu, 1999): Es un instrumento de autoaplicación que evalúa el autoconcepto en cinco dimensiones (Académicolaboral, Social, Emocional, Familiary Físico) y consta de 30 ítems que se miden en una escala Likert de cinco puntos quevan desde "Nunca" (1) hasta "Siempre" (5). La validez se llevó a cabo mediante Análisis Factorial Exploratorio y la confiabilidad mediante la prueba Alfa de Cronbach $(\alpha=.81)$.

\section{Procedimiento}

Para la realización de este estudio, primero se solicitó el permiso correspondiente a las autoridades del albergue para la aplicación de los instrumentos. Para ello se coordinó con el Departamento de Psicología de la institución. Se procedió a informar los lineamientos de la investigación mediante un asentimiento informado entregado a las participantes, se garantizó la confidencialidad de los datos y se mencionó que la participación era totalmente voluntaria. Para el análisis de los datos, se utilizó el programa estadístico Jamovi Versión o.9.2.3, haciendo uso del coeficiente de correlación de Pearson.

\section{Resultados}

Como se observa en la Tabla 1, existe una correlación estadísticamente significativa, directa y con un tamaño del efecto grande entre la resiliencia y el autoconcepto $(\mathrm{r}=.789, \mathrm{p}<$.001). Asimismo, se aprecian correlaciones directas y moderadas entre la resiliencia y las dimensiones del autoconcepto; del mismo modo, se encuentran correlaciones directas y moderadas entre el autoconcepto y las dimensiones de la resiliencia.

Tabla 1. Matriz de correlación entre la resiliencia y el autoconcepto

\begin{tabular}{|lcccccc|}
\hline & Autoconcepto & Académico-laboral & Social & Emocional & Familiar & Físico \\
\hline Resiliencia & $.789^{* * *}$ & $.516^{* *}$ & $.614^{* * *}$ & $.596^{* * *}$ & $.530^{* *}$ & $.562^{* * *}$ \\
Insight & $.542^{* *}$ & $.395^{*}$ & $.391^{*}$ & $.400^{*}$ & $.376^{*}$ & $.380^{*}$ \\
Independencia & $.709^{* * *}$ & $.493^{* *}$ & $.529^{* *}$ & $.612^{* * *}$ & $.394^{*}$ & $.478^{* *}$ \\
Interacción & $.52^{* *}$ & .256 & $.599^{* * *}$ & $.449^{* *}$ & $.384^{*}$ & .263 \\
Moralidad & $.760^{* * *}$ & $.587^{* * *}$ & $.516^{* *}$ & $.562^{* * *}$ & $.430^{*}$ & $.619^{* * *}$ \\
Humor & $.465^{* *}$ & .187 & $.479^{* *}$ & $.370^{*}$ & $.408^{*}$ & .218 \\
Iniciativa & $.645^{* * *}$ & $.577^{* * *}$ & $.393^{*}$ & $.375^{*}$ & $.362^{*}$ & $.619^{* * *}$ \\
Creatividad & $.680^{* * *}$ & .346 & $.489^{* *}$ & $.532^{* *}$ & $.566^{* * *}$ & $.518^{* *}$ \\
\hline
\end{tabular}

Nota: ${ }^{*} \boldsymbol{p}<.05,{ }^{* *} p<.01,{ }^{* * *} p<.001$ 


\section{Discusión}

Las adolescentes en situación de abandono, debido a los acontecimientos vitales estresantes que han vivido, pueden verse afectadas a nivel psicológico. A pesar de ello, su pronóstico no necesariamente es negativo, pues los mismos eventos adversos las impulsan a generar sus propios recursos para lidiar con ello (Drapeau et al., 2007; Samuels \& Pryce, 2008). En tal sentido, la presente investigación tuvo como principal objetivo determinar la relación entre la resiliencia y el autoconcepto.

Tal como se muestra en los resultados, existe una relación directa y moderada entre ambas variables, lo que coincide con las investigaciones de Honey et al. (2011) y Kurniawan et al. (2018). Esto podría deberse a cuatro factores importantes presentados a continuación:

En primer lugar, al ser identificada la situación de vulnerabilidad, las adolescentes son separadas de su entorno familiar y llevadas a un centro de acogida como una medida de protección, lo cual implica cortar lazos con personas significativas para ellas. Esto les afecta a nivel físico, cognitivo, conductual, social y les genera un fuerte deseo de no querer estar en ese lugar ni continuar viviendo una situación desagradable; por lo que se dan cuenta de su realidad y se motivan a actuar para cambiarla (Drapeau et al., 2007; Samuels \& Pryce, 2008).

En segundo lugar, al estar motivadas a cambiar y reconocer que se ha interrumpido la interacción con sus figuras parentales, comienzan a entablar nuevos vínculos significativos con otras personas como cuidadores, trabajadores sociales, profesores y compañeros de clase, generando nuevas redes de soporte (Drapeau et al., 2007; Edmond et al., 2006). Esto permite que las adolescentes reciban una retroalimentación positiva de su entorno y perciban un mejor desempeño de sí mismas a nivel social. Es interesante observar que la resiliencia presenta la correlación más alta con el autoconcepto social, confirmando así que el hecho de sentirse integradas y valoradas por su entorno, incrementa sus recursos para sobrellevar la adversidad (Kurniawan et al., 2018). En consecuencia, se podría afirmar que la dimensión relacional parece ser más importante en esta población, pero paradójicamente, el mismo hecho de estar institucionalizadas en un albergue, limita su acceso o cantidad de apoyo social (Edmond et al., 2006).

En tercer lugar, las adolescentes suelen participar de actividades académicas, culturales o talleres productivos, donde además de socializar, tienen la oportunidad de descubrir y desarrollar nuevas habilidades. Estas se incentivan a través de reconocimientos materiales o sociales, reforzando así su autoconcepto, la sensación de control y de responsabilidad, ya que pueden sentirse como un modelo para el resto de adolescentes del albergue (Drapeau et al., 2007).

En cuarto lugar, cuando las adolescentes institucionalizadas obtienen logros a nivel académico, además de tener experiencias 
positivas con maestros y compañeros, se perciben como personas valiosas para su entorno y con mayor control de su vida, por lo que se planifican mayor cantidad de metas profesionales a futuro (Edmond et al., 2006). Por ello, este éxito a nivel escolar contribuye a la mejora del autoconcepto y la resiliencia, pues les da mejores herramientas para lograr su autonomía y superar su situación actual (Höjer \& Johansson, 2013; Honey et al., 2011; Samuels \& Pryce, 2008; Shavelson \& Bolus, 1982).

En conclusión, las adolescentes institucionalizadas por abandono, perciben esta situación como un reto a afrontar (Samuels \& Pryce, 2008), que las ha llevado a desarrollar estrategias de afrontamiento como vincularse con nuevas figuras de referencia, quienes les dan soporte y retroalimentación positiva en el aspecto social y académico, lo cual contribuye al incremento del autoconcepto, que a su vez, les da una sensación de mayor competencia, motivos para superarse, un propósito de vida y expectativas respecto a su futuro (Domhardt et al., 2015; Kurniawan et al., 2018).

Cabe señalar que el presente estudio no es concluyente dado que tiene ciertas limitaciones, entre ellas, la muestra no fue tomada aleatoriamente. Por otro lado, esta investigación considera la relación entre dos variables por lo cual es recomendable que futuros estudios tengan en cuenta una cantidad muestral más amplia y seleccionada de forma probabilística, así como considerar otras variables importantes en el estudio de la resiliencia en esta población, pues puede haber muchos otros factores que también tengan relación con esta variable de estudio.

Finalmente, en base a los hallazgos, sugerimos que las intervenciones para mejorar la resiliencia y el autoconcepto de esta población se centren en el aspecto social, a través de la incorporación en eventos académicos, culturales, deportivos y en la promoción de espacios de interacción con sus pares.

\section{Financiamiento}

La presente investigación fue autofinanciada.

\section{Conflictos de interés}

Los autores declaran que no tienen conflictos de interés. 


\section{Referencias}

Chua, L. W., Milfont, T. L., \& Jose, P. E. (2015). Coping Skills Help Explain How Future-Oriented Adolescents Accrue Greater Well-Being Over Time. Journal of Youth and Adolescence, 44(11), 2028-2041. https://doi.org/10.1007/s10964-014-0230-8

Cox, P., \& Shore, H. (2017). Absent Fathers and Family Breakdown: Delinquency in Vichy France. En Becoming Delinquent: British and European Youth, 1650-1950 (pp. 153-170). Routledge.

Crocetti, E. (2017). Identity Formation in Adolescence: The Dynamic of Forming and Consolidating Identity Commitments. Child Development Perspectives, 11(2), 145-15o. https://doi.org/10.1111/cdep.12226

Crocetti, E., Rubini, M., Branje, S., Koot, H. M., \& Meeus, W. (2016). Self-Concept Clarity in Adolescents and Parents: A Six-Wave Longitudinal and Multi-Informant Study on Development and Intergenerational Transmission. Journal of Personality, 84(5), 580-593. https://doi.org/10.1111/jopy.12181

Defensoría del Pueblo. (2011). Niños, niñas y adolescentes en abandono: aportes para un nuevo modelo de atención.

Denton, E. D., Musa, G. J., \& Hoven, C. (2017). Suicide behaviour among Guyanese orphans: identification of suicide risk and protective factors in a low- to middle-income country. Journal of Child \& Adolescent Mental Health, 29(3), 187-195. https://doi.org/10.2989/17280583.2017.1372286

Dey, P., \& Beena Daliya, R. (2019). The Effect of Resilience on the Psychological Well Being of Orphan and Non-Orphan Adolescents. Indian Journal of Mental Health, 6(3).

Domhardt, M., Münzer, A., Fegert, J. M., \& Goldbeck, L. (2015). Resilience in Survivors of Child Sexual Abuse. Trauma, Violence, E Abuse, 16(4), 476-493. https://doi. org/10.1177/1524838014557288

Drapeau, S., Saint-Jacques, M.-C., Lépine, R., Bégin, G., \& Bernard, M. (2007). Processes that contribute to resilience among youth in foster care. Journal of adolescence, 30(6), 977-999. https://doi.org/https://doi.org/10.1016/j.adolescence.2007.01.005 
Edmond, T., Auslander, W., Elze, D., \& Bowland, S. (2006). Signs of Resilience in Sexually Abused Adolescent Girls in the Foster Care System. Journal of Child Sexual Abuse, 15(1), 1-28. https://doi.org/10.130o/Jo7ov15no1_o1

García, F., \& Musitu, G. (1999). Autoconcepto forma 5. TEA.

Gobierno Regional de Cajamarca (GRC). (2017). Plan Estratégico Institucional 2016 - 2019.

Höjer, I., \& Johansson, H. (2013). School as an opportunity and resilience factor for young people placed in care. European Journal of Social Work, 16(1), 22-36. https:// doi.org/10.1080/13691457.2012.722984

Honey, K. L., Rees, P., \& Griffey, S. (2011). Investigating self-perceptions and resilience in Looked After Children. Educational Psychology in Practice, 27(1), 37-52. https:// doi.org/10.108o/02667363.2011.549352

Humphreys, K. L., Miron, D., McLaughlin, K. A., Sheridan, M. A., Nelson, C. A., Fox, N. A., \& Zeanah, C. H. (2018). Foster care promotes adaptive functioning in early adolescence among children who experienced severe, early deprivation. Journal of Child Psychology and Psychiatry, 59(7), 811-821. https://doi.org/10.1111/jcpp.12865

Jaffee, S. R., Caspi, A., Moffitt, T. E., Polo-Tomás, M., \& Taylor, A. (2007). Individual, family, and neighborhood factors distinguish resilient from non-resilient maltreated children: A cumulative stressors model. Child Abuse E Neglect, 31(3), 231-253. https://doi.org/10.1016/j.chiabu.2006.03.011

Kurniawan, B., Neviyarni, N., \& Solfema, S. (2018). The relationship between self-esteem and resilience of adolescents who living in orphanages. International Journal of Research in Counseling and Education, 1(1), 47-52.

Masten, A. S. (2011). Resilience in children threatened by extreme adversity: Frameworks for research, practice, and translational synergy. Development and Psychopathology, 23(2), 493-506. https://doi.org/10.1017/So954579411000198

McGloin, J. M., \& Widom, C. S. (2001). Resilience among abused and neglected children grown up. Development and Psychopathology, 13(4), 1021-1038. https:// doi.org/10.1017/So95457940100414X

Ministerio de la Mujer y Poblaciones Vulnerables (MIMP) (2014). Sistemas de Protección de Derechos de las Niñas, Niños y Adolescentes. 
Mishra, R., \& Sondhi, V. (2019). Fostering Resilience among Orphaned Adolescents through Institutional Care in India. Residential Treatment for Children \& Youth, 36(4), 314-337. https://doi.org/10.108o/o886571X.2018.1535286

Moneta, G. B., Schneider, B., \& Csikszentmihalyi, M. (2001). A Longitudinal Study of the Self-Concept and Experiential Components of Self-Worth and Affect Across Adolescence. Applied Developmental Science, 5(3), 125-142. https://doi.org/10.1207/ S1532480XADSo503_2

Morris, A. S., Criss, M. M., Silk, J. S., \& Houltberg, B. J. (2017). The Impact of Parenting on Emotion Regulation During Childhood and Adolescence. Child Development Perspectives, 11(4), 233-238. https://doi.org/10.111//cdep.12238

Musitu, G., \& García, J. F. (2004). Consecuencias de la socialización familiar en la cultura española. Psicothema, 16(2), 288-293.

Oshri, A., Carlson, M. W., Kwon, J. A., Zeichner, A., \& Wickrama, K. K. A. S. (2017). Developmental Growth Trajectories of Self-Esteem in Adolescence: Associations with Child Neglect and Drug Use and Abuse in Young Adulthood. Journal of Youth and Adolescence, 46(1), 151-164. https://doi.org/10.1007/s10964-016-0483-5

Papalia, D. E., Feldman, R. D., \& Olds, S. W. (2009). Human development. McGraw-Hill.

Prabandani, L., \& Santoso, H. P. (2017). The Correlation between The Intensity of Broken Home Family Communication and Peer Group Interaction with Adolescent's Self Concept. Interaksi Online, 21(1), 1-14.

Prado, R., \& Del Águila, M. (200o). Estructura y funcionamiento familiar en adolescentes resilientes y no resilientes. Universidad Federico Villarreal.

Programa Integral Nacional para el Bienestar Familiar (INABIF) (2019). Boletín Mensual $N^{\circ} 10$.

Rew, L., Taylor-Seehafer, M., Thomas, N. Y., \& Yockey, R. D. (2001). Correlates of Resilience in Homeless Adolescents. Journal of Nursing Scholarship, 33(1), 33-40. https://doi.org/10.1111/j.1547-5069.2001.00033.x

Rowe, S. L., Zimmer-Gembeck, M. J., \& Hood, M. (2016). Community, Family, and Individual Factors Associated with Adolescents' Vulnerability, Daily Stress, and Well-being Following Family Separation. Journal of Divorce \& Remarriage, 57(2), 87-111. https://doi.org/10.1080/10502556.2015.1127875 
Rutter, M. (1999). Resilience concepts and findings: implications for family therapy. Journal of Family Therapy, 21(2), 119-144. https://doi.org/10.1111/1467-6427.00108

Samuels, G. M., \& Pryce, J. M. (2008). "What doesn't kill you makes you stronger": Survivalist self-reliance as resilience and risk among young adults aging out of foster care. Children and Youth Services Review, 30(10), 1198-1210. https://doi. org/10.1016/j.childyouth.2008.03.005

Santoso, S., \& Zamroni, E. (2017). Analysis of Social and Emotional Development of Orphaned Youth in terms of Self Concept and Resilience: Study at Child Care Children's Home (PSAA) Tunas Bangsa Pati. GUIDENA: Jurnal Ilmu Pendidikan, Psikologi, Bimbingan dan Konseling, 7(1), 87-93.

Shavelson, R. J., \& Bolus, R. (1982). Self concept: The interplay of theory and methods. Journal of Educational Psychology, 74(1), 3-17. https://doi.org/10.1037/oo22-0663.74.1.3

Shavelson, R. J., Hubner, J. J., \& Stanton, G. C. (1976). Self-Concept: Validation of Construct Interpretations. Review of Educational Research, 46(3), 407-441. https:// doi.org/10.3102/00346543046003407

Sherr, L., Roberts, K. J., \& Croome, N. (2017). Emotional distress, resilience and adaptability: a qualitative study of adults who experienced infant abandonment. Health Psychology and Behavioral Medicine, 5(1), 197-213. https://doi.org/10.108 o/21642850.2017.1297238

Smith, C. A., Park, A., Ireland, T. O., Elwyn, L., \& Thornberry, T. P. (2013). Long-Term Outcomes of Young Adults Exposed to Maltreatment. Journal of Interpersonal Violence, 28(1), 121-156. https://doi.org/10.1177/o886260512448845

Woods, S. B., Farineau, H. M., \& McWey, L. M. (2013). Physical health, mental health, and behaviour problems among early adolescents in foster care. Child: Care, Health and Development, 39(2), 220-227. https://doi.org/10.1111/j.1365-2214.2011.01357.x

Zolkoski, S. M., \& Bullock, L. M. (2012). Resilience in children and youth: A review. Children and Youth Services Review, 34(12), 2295-2303. https://doi.org/10.1016/j. childyouth.2012.08.009

Recibido: 30 de setiembre de 2020

Revisado: 7 de enero de 2021

Aceptado: 16 de julio de 2021 